\title{
Automatic personalized interventions for behaviour change towards healthier lifestyle
}

\author{
Inmaculada Luengo López \\ Head of R\&D department \\ Hi-Iberia Ingeniería y Proyectos \\ Madrid, Spain \\ iluengo@hi-iberia.es
}

\author{
Paloma Jimeno Sánchez-Patón \\ Scientific Coordinator \\ Hi-Iberia Ingeniería y Proyectos \\ Madrid, Spain \\ pjimeno@hi-iberia.es
}

\author{
Pablo Aubert Girbal \\ Head of Software Development \\ Hi-Iberia Ingeniería y Proyectos \\ Madrid, Spain \\ paubert@hi-iberia.es
}

Received: July 20, 2021. Revised: August 30, 2021. Accepted: September 3, 2021. Published: September 9, 2021.

\begin{abstract}
This paper outlines the results evidenced by WellCo (GA no: 769765), an European project funded by the European Commission within its $\mathbf{H 2 0 2 0}$ programme under the personalised medicine call. The aim of this project was to develop and validate how ICT technologies may engage people to adopt healthier behaviour choices that improve their wellbeing status for as long as possible.
\end{abstract}

Using data from wearable devices and AI-based algorithms, WellCo assesses the status of the user in terms of wellbeing and the risk of CVD. Using this information, WellCo develops an affective-aware coach that empowers users in the process of change of behaviour through the provision of interventions tailored to their current $\operatorname{mood}$ and life context. These motivational activities ranged from recommendations, goals to achieve, interactions with people in the social network, tips from experts and supporting groups suggested by the platform and adapted to their needs.

The project has been validated with ageing people in Italy, Denmark and Spain. Despite the COVID-19 situation, results are very promising in terms of the possibilities that ICT technologies have for health promotion and set the basis for further research in this direction.

Keywords- behaviour, capability, change, healthy, motivation, opportunity, coach, wellbeing

\section{INTRODUCTION}

Europe and the rest of the world is living a period of volatility and uncertainty with several underlying trends conditioning the action of the economical, societal and political agents: pandemics, ageing society, inequality, climate change, etc. These trends pose important challenges and highlight the importance of postulating societal shifts that constitute a deep break with the past.

One of the main challenges is related to public spending on healthcare and how to ensure long-term sustainability of social welfare and healthcare systems, especially in a scenario of ageing population. In this context, the digitalization and health promotion policies play a key role and constitute one of the greatest challenges of healthcare environment, with important challenges at structural and cultural level [1].

The digital transformation of health care can be disruptive; technologies such as the Internet of Things, virtual care, remote monitoring, artificial intelligence, big data and smart wearables have proven potential to create continuum of care and reducing the gap between the demand and the quantity and quality of the supply of healthcare. In this sense, the COVID-19 pandemic seems to have demonstrated the main challenges and needs in the health system and have accelerated the changes that will enable to reach this digital transformation and, therefore, the complete incorporation of personalized and accurate medicine [2].
Secondly, a sustainable, efficient and cost-effective future for health also requires a shift from the culture of disease to the culture of prevention and health promotion. This is quite challenging and involves changes in all areas of society, both at educational, political, cultural and economic level. In the coming years, it is expected that public policies tax unhealthy behaviours, that companies award to those employees that have healthier habits (e.g. paying if cycling instead of using the car) [3] and that there is a huge demand of applications for digital health promotion and literacy [4].

In this sense, several new ICT based concepts and initiatives aimed at empowering, coaching and training people to change behaviours through the design of tailored motivational interventions have arisen in the latest years.

To determine the type of intervention that fits the best for each type of user, most of these initiatives leverage in recommender systems. These systems are used to provide feedback and recommendations on health status and health behaviours, such as lifestyle [5], nutrition [6], obesity [7], diabetes [8][9], drug side effects [10] and smoking cessation [11][12]. They leverage contextual data about users (location, profile information, data from smart wearables, electronic health records, etc.) to predict the type of intervention needed. Thus, in order to allow recommender systems to be effective, precise and accurate AI algorithms to assess the status, capabilities and motivation of the user are needed.

Emerging wearable devices, intended to monitor the physiological parameters of users, and the growth of machine learning technologies, applied to predict the risk of disease, have enable the development of great variety of algorithms aimed at assessing the general status of patients, the risk of developing a given disease condition and the main factors affecting this risk - key elements for the provision of recommendations that support health promotion. The set of non-communicable disease covered by these algorithms range from stroke risk prediction [13], CVD risk [14], type 2 diabetes risk[15] and cancer [16].

However, being able to extract risk patterns and the probability of suffering from a certain condition, is not enough to empower people to change behaviours and to foster health promotion. Recommendation systems have also to address the capability and motivation of the user to change a certain behaviour and being able to guide and follow-up people during the whole behaviour change process.

Because of that, WellCo is not just about the provision of tailored recommendations, but it centres the provision of behaviour interventions on the behaviour change wheel [17], a method for characterising and designing behaviour change actions that addresses essential conditions such as capability, 
opportunity and motivation (what it is termed the "COM-B" system) when providing recommendations.

In addition, to keep users motivated, WellCo offers an affective-aware virtual coach that continuously advice, train and guide the user in the process of adoption of healthier daily life habits that could reduce the risk of onset of disease in the long term.

WellCo system is based on the behaviour change wheel [17], a new method for characterising and designing behaviour change actions around three essential conditions: capability, opportunity and motivation (termed the "COMB" system). Taking this into consideration, the flow for behaviour change in WellCo is divided into three phases:

- Understanding the behaviour and context of people;

- Personalize interventions in such a way that they fit the capabilities and opportunities of people;

- Motivate people towards behaviour change through social incentives, immediate rewards, progress monitoring and continuous support.

Along this paper, the behaviour change process proposed by WellCo will be analyzed. WellCo consists of three applications:

- the main app for elderly people that identifies the context of the user and provides the recommendations towards behaviour change,

- the app for friends and informal caregivers that enable them to motivate elderly users by participating in the social network that WellCo provides,

- the web app for clinicians that enable experts to check the status of users, share valuable content related to wellbeing and validate recommendations.

The paper is structured as follows:

Section II WellCo Methodology tries to describe the architecture and main elements driving the behaviour change model proposed. Section III analyses the main results derived from the qualitative and quantitative evaluation carried out after the validation of WellCo with 93 users from Italy, Denmark and Spain in trials with a duration of 4 months. Section IV offers a reflexion over the implementation of WellCo, the results obtained and the impact that COVID had on the project.

\section{Wellco Methodology}

\section{A. Understanding the behaviour of people}

The emergence of wearable devices of the latest years has presented an exciting opportunity to monitor human physiological parameters in a continuous, real-time and nonintrusive manner [18]. These devices are relevant as they enable to extract patterns of behaviour but also other contextual data, such as weather, that could be relevant when understanding these behaviours. WellCo have used three types of commercial wearable devices to monitor the behaviour of users: Withings Steel $\mathrm{HR}^{1}$, Mobvoi Ticwatch E2 and Motorola E6 Play. The two first ones are smartwatches while the latest is the smartphone over which WellCo application was installed.

From the smartwatches the following information was extracted: steps, heart rate, calories, physical activity and sleep patterns. In the case of the Ticwatch, raw data from accelerometers was also considered. For the case of the smartphone, the former data was accessed: accelerometers, gyroscope, magnetometer, Wifi access points nearby and categories of apps in the foreground.

Also, in order to get more insights about the patterns of behaviour of users, WellCo also leverages scientifically validated questionnaires to get trends of users in relation to physical activity[19], nutrition, sleep[20], general health[21], mental status[22][23], etc.

Finally, the front camera of the smartphone and the microphone are used to monitor the emotional status of the user. When the user interacts with the smartphone, the speech emotion classifier[24] analyses the emotions of the user based on his/her voice while, simultaneously, the emotion classifier based on visual features, processes the emotional status of the user by using face movements when interacting with the coach. Both classifiers are part of a metamodel for emotion classifier that learns to weight the outputs of every single classifier. To train this metamodel, a dataset with 4 users from Italy and 4 for Spain was created. The dataset showed 39 scenarios per user where they pretended emotions, both using voice and facial expression [25]. When training the model, Random-Forest machine learning and the leave-one-subject-out approach was used, meaning that the features for three users were taken for training, and the last user was used to test the performance of the meta-model. This process was repeated for four times. The features that were used for training the meta-model were the probabilities that each of the individual models returned of a sample containing anger, sadness, happiness and neutral emotion. Thus, a total of eight features were provided as input to the meta-model (four probabilities from the speech single classifier, and four probabilities from the visual one).

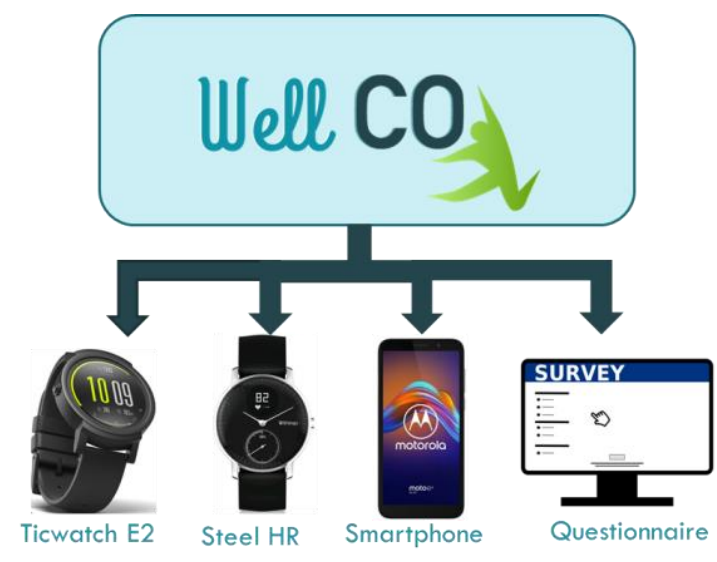

Fig. 1. Devices used in WellCo

After defining the main inputs considered in WellCo, the next step was defining how these inputs are processed in order to understand the behaviour of users and the areas where they need to improve to promote their health status. 
There are different modules in WellCo aimed at analysing this input data. Concretely, there are:

- Mental: based on an ML model that detects episodes of stress, anxiety and depression[26] using as input wearable devices and the scores from the Patient Health Questionnaire (PHQ-4) and the Perceived Stress Scale 4 (PSS-4).

- Nutrition: that determines the type of meal (small, medium or big meal) based on the bite counting algorithm developed using raw data from Ticwatch[29][30] as well as the nutrition habits of the user based on the validated questionnaires [31][32].

- Physical Activity and Sleep: that analyses data coming from sleep, physical activity and energy expenditure to get insights about the pattern of activity of the user[33][34].

Now that WellCo has an assessment of the status of the user and the main variables where improvement or action is needed, the next step is to provide the recommendations to user and to personalize these interventions so they meet the capability and opportunity of the user to adopt them.

\section{B. Personalize interventions}

Along this section, the main focus will be on the way in which WellCo provides recommendations to users and how these recommendations are personalized so they properly address the capabilities of users.

WellCo counts on different recommenders covering the main areas around healthcare where the user could need support: nutritional recommender, wellbeing recommender, mental recommender, social recommender, emotional recommender and general recommender. Each of these recommenders is activated or not based on the variables impacting the wellbeing of the user. The output are basic recommendations that are later personalized and provided to users through the WellCo coach. Recommendations are always supervised by experts who will be the ones deactivating those recommendations that according to their criteria are not useful or could be harmful for the user. Following, a detailed description of the main recommenders included in WellCo is provided.

In the first place, the wellbeing recommender has been developed over a Rule Management Ontology that supports the representation of event-based rules that trigger specific actions based on the impact factors related to physical activity and sleep. The ontology that constitutes the base of this recommender has been implemented in Protégé v5.0, a free, open-source ontology editor and framework for building intelligent systems. This ontology has been built on the bases of the UniversAAL ontologies that were edited by PM-15 Nestore Project[36] and that are accessible in the Nestore Gitlab repository ${ }^{2}$. For the case of the wellbeing recommender, the ontology has been built on the bases of the sleep quality and physical activity behaviour ontology. Rules in the form of sleep and physical activity have been defined on the basis of scientific literature and research. To perform reasoning and infer new knowledge that enable the recommender to generate more information, a Pellet reasoner have been defined on the bases of the predefined SWRL rules.

Secondly, the social recommender has been implemented as a neighbourhood-based recommendation system using graphs. These recommender systems are characterized because they are able to capture local associations in the data, consequently, it is possible to recommend to a user an item that it is very different from his/her usual taste or an item that it is not well known, if one of his closest neighbours has given it a strong rating. Although this recommendation may not be a guaranteed success, it may help the user to discover a whole new item. If we define an "item" as a WellCo user that could potentially be a friend of a certain user, it can be extracted that this type of recommenders are the most suitable for the social network in WellCo. Thus, it will allow the user to receive suggestions of friendship with users that are very different from the user's usual profile of friends and, in this way, expand his/her level of social interaction. To assess the similarity between users, every user node in the graph is represented according to the country, profession and kind of pet (in case the user has).

In order to avoid overwhelming the user with suggestions of friendship and also because of the limited number of users that WellCo already has, the Social Recommender is triggered once a week (more specifically on Sundays) and only 2 users are suggested per interaction.

The emotional recommender is fed with the emotion of the user extracted by the metamodel for emotional analysis when the user interacts with the coach. In case emotions with a negative polarity are detected, e.g. "Angry" or "Sad", a set of recommendations are immediately provided by the coach in order to change the mood of the senior.

Finally, the general recommender provides daily recommendations and tips to users with the purpose of reminding them the importance of staying healthy. These recommendations do not consider the results from the assessment of the user but they consist on daily preventive activities that are beneficial for the wellbeing of the user.

Thus, at this point, the main outputs of provision of healthier interventions to users have been described. However, these interventions will be not effective unless they are framed in the context and capability of the user. In this sense, one of the main factors making that the proposed behaviour change actions are successful is the personalization.

In relation to personalization, it is important to highlight that the recommendations are provided based on the assessment of the user and the main factors impacting their health. Moreover, the recommendations are customized to the user so they are aligned with the context surrounding him/her: name, if rural/urban, if pet, if children, etc. For example, if we want the user to take more steps and we know that the user has a dog, instead of providing a recommendation of taking 5,000 steps, the recommendation includes something like: "Walk with your dog every day for 30 minutes". Also, all the recommendations have the name of the user so he/she feels that this recommendation has been created just for him/herself. 


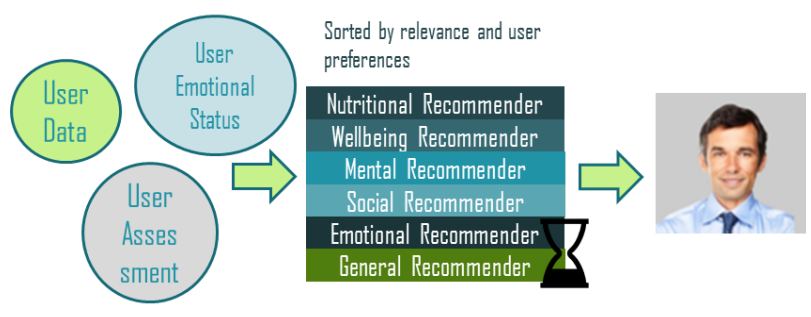

Fig. 2. Recommenders in WellCo

Finally, all the recommendations are provided to the user through the effective-aware coach. The coach uses data from the emotion detection to understand the emotional status of the user and show empathy when providing these recommendations. In addition, the information exchanged with the coach when the user interacts with it, is used to get more knowledge about the user and being able to provide more personalized recommendations. All these features are explained in further detail in the section below related to motivation of the user.

\section{Motivation of people}

Along the previous sections, it has been reviewed the process that WellCo follows in order to understand the status of the user and provide personalized interventions that fit the capability and opportunity/context surrounding the user. However, all this effort is meaningless, if the necessary tools are not available to motivate the user and ensure the adoption of these interventions by him/her in the medium and long term. Thus, motivation is the core of the behaviour change process that WellCo proposes.

The main motivational element of WellCo is the affective-aware virtual coach. This Embodied Conversation Agent (ECA) named as "virtual coach" aims to serve as novel modality for the delivery of behaviour change interventions. The coach delivers personalized recommendations to users with the purpose of guiding and motivating them in the process of behaviour change. These interventions focus on all areas affecting the wellbeing status of a certain user (nutrition, physical activity, mental status, etc) and take into consideration both the preferences of the user and prioritization of actions. See section B for more details.

This coach implemented in WellCo has anthropomorphic features corresponding to humans and based on this, three representations, man, woman and cartoon, are available. So the user is able to select the representation with which he/she feels most identified. The coach representation in WellCo has been designed and implemented according to the features provided by the SitePal library ${ }^{3}$, a framework for creating and customizing web avatars.

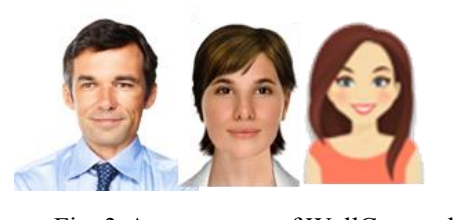

Fig. 3. Appearances of WellCo coach

The coach delivers verbal communication via speech and text to the user, the former with lips synchronization so the user perceives the coach with human features, interacting

\footnotetext{
${ }^{3}$ https://www.sitepal.com/
}

with it in a more natural way. However, in addition to verbal communication, nonverbal communication also plays an important role in the coach implemented in WellCo. On the basis of the metamodel for emotion analysis mentioned above, the user's facial expressions and features of speech are captured and processed in an attempt to assess the user's most probable emotional status and thus, convey an ongoing sense of empathy via the coach-based interface. The set of emotions to be shown by the coach as well as their intensity have been adapted based on the possibilities that the own SitePal library provides.

In order to motivate the user on the path towards behaviour change the virtual coach, in addition to the provision of recommendations, is also able to keep conversations with the user. This conversation is created in such a way that allows the user to perceive the coach as a colleague and to build trust with it.

In order to achieve this, every time that the user starts a conversation with the coach, all the information about the user that is stored in the database is charged. Thus, when the user interacts with the coach, it seems that the coach knows the user (children name, hobbies, if married, pet name, daily activities, etc). In fact, during the conversation, the coach also extracts information from the user that is stored in the database at the end of the conversation and used to personalize recommendations. For example, during the conversation the coach could ask the user about the name of your pet and update the database with this information. In this way, when there is a recommendation, there is the possibility to know that the user has a pet and the name of this pet so it is possible to provide a fully personalized recommendation.

The coach has been implemented as a rule based chatbot using AIML language. These chatbots are characterized because they use rules pattern-template, where pattern defines the pattern to match what a user may input to the coach and template defines the response of the chatbot to user's input. The pattern is associated with one or more keywords that could happen in the sentence of the user input. In the speaking time of the coach, the coach answers with the template associated to the keywords that has the highest score in relation to the declaration made by the user. In the case that the chatbot does not have any template that match the input of the user, it could provide something like "It is very interesting" or even to propose a conversation about something that the coach knows about the user: "Do you have any plan with your grandchildren Alfred?".

However, despite the previously mentioned, it was clear that not all the burden of motivation could be on the coach. Because of that, it was understood that other elements aimed at encouraging the user in the adoption of healthier behaviours were considered in WellCo. Doctor Tali Sharot, neuroscientist at University Colleage London, states that to induce behaviour change actions is not enough with providing recommendations about healthier activities or to warn people about the consequences that maintaining behaviours in the long term could have. To ensure successful behaviour change actions, motivation has to be built on the basis of three pillars: social incentives, immediate rewards and progress monitoring and continuous support[38].

Thus, following these guidelines, the whole motivational flow of behaviour change in WellCo was completed. 
In terms of social incentives, Goals and the Social Network play a relevant role. On the one hand, recommendations can be set as goals for the user to achieve. Every time that a user in the Social Network achieves a goal, friends of this user are notified about the goal and they can make comments, send likes, etc. Thus, notifications are a way to incentive friends of this user to also achieve their goals. Also, the reaction of friends to these goal notifications can be seen as immediate rewards for users.

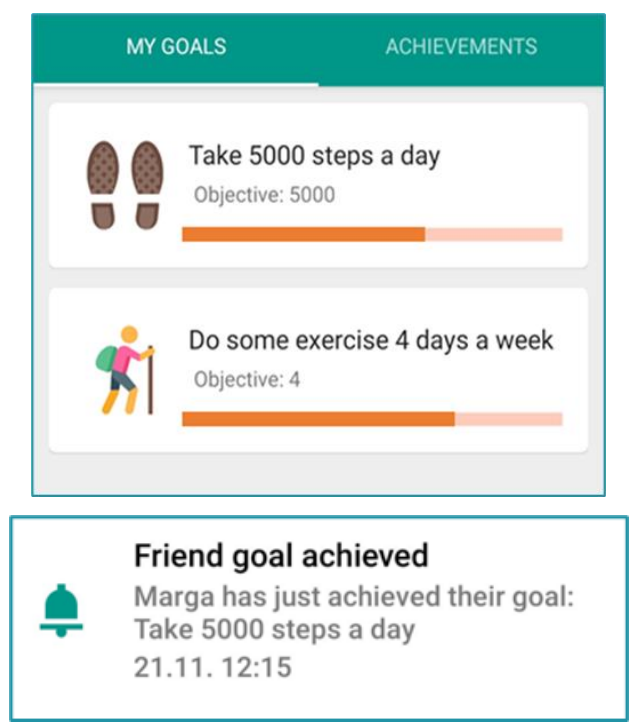

Fig. 4. Goals visualization and goals notification in WellCo

Following in the wake of motivation, WellCo also provides users with tools for progress monitoring and continuous support.

In terms of progress monitoring, users can access historical data about steps, sleep, heart rate and weight to see their progress around wellbeing and keep improving.

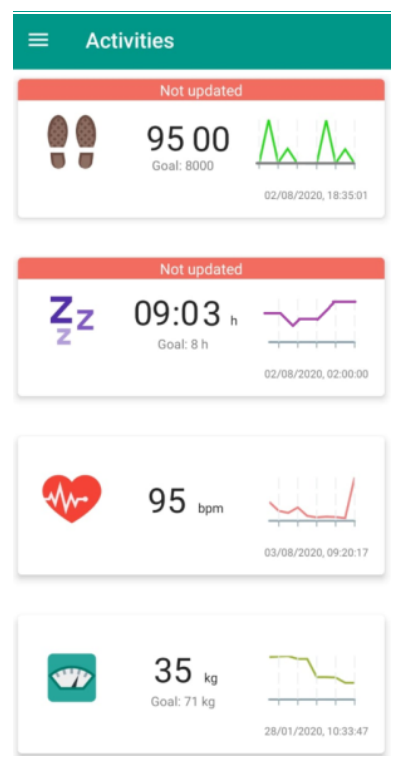

Fig. 5. Activities Section

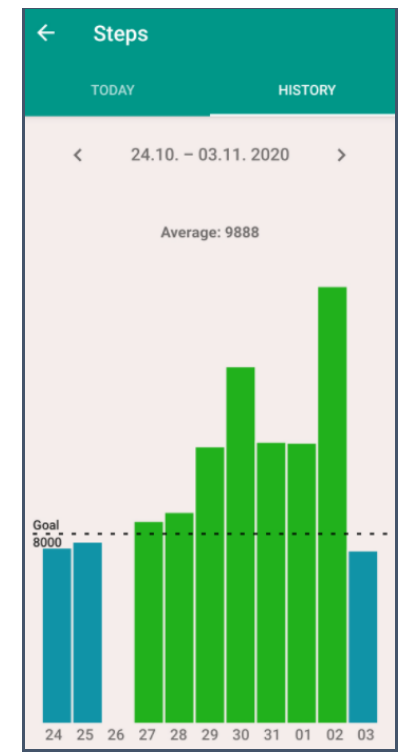

Fig. 6. Steps Overview
For continuous support, there are different elements to help the user to feel motivated and guided.

On the one hand, the virtual coach is continuously available with the provision of recommendations and with special support about certain wellbeing areas if needed.
On the other hand, the platform of the expert catches the most common profiles of users (men, living in rural area, with children, risk factors, etc) and automatically suggests the expert the creation of video-groups where inviting people with such profile to discuss about it. This video-groups act as supporting groups so users can know people with the same circumstances they have and join effort to overcome this situation and keep healthy.

Finally, experts can create videos, documents, etc addressing different areas around wellbeing: nutrition, physical activity, mental health, etc and sharing this material with all or certain users in the WellCo platform so there is sufficient material available for users to keep moving forward on the path towards wellbeing.

\section{EVALUATION AND RESULTS}

This section aims at presenting the process and results derived from the validation of the WellCo from October to January 2021.

The sample was composed by 93 users aged among 55 and more from rural and urban areas of the three European countries participating in the project (Italy, Denmark and Spain). The characteristics of participants from every country are the following:

In Spain, users for the trial were recruited in a rural environment aged over 65, mainly living alone or with informal caregivers in some cases. In addition, most of them had multiple pathologies and a certain level of fragility. A total of 40 users were involved.

In Denmark and Italy, users came from urban environment and they were between 55 and 65 years in the case of Denmark and over 65 in Italy. They were healthy albeit with health-risk issues. A total of 40 users were involved in Italy and 13 users in Denmark. The reason why the sample of users was more reduced in Denmark was that the recruitment process was made online (without face-toface meetings) and most users were reluctant to participate.

Besides, some exclusion criteria were applied, such as people with cognitive difficulties and/or mental or personality disorders diagnosed because they were unable to decide for themselves if they liked to participate; blind or deaf people were not included due to this condition determine the inability and/or unfeasibility of following the WellCo recommendations. Besides, minimum skills of handling new technologies and daily access to wi-fi connection were preferable.

Each of these participants received a smartphone (where they had the WellCo app installed) and a smartwatch (Withings Steel HR) for validation purposes.

Informal caregivers and professionals (like social workers or general practitioners) participated in the project as element to motivate users. In the case of informal caregivers, this motivation came from the Social Network while in the case of professionals was derived from the validation of recommendations, the creation of supporting groups and the provision of material related to health and wellbeing.

The main outcome of this validation was to show how the process towards behavior change proposed by WellCo was able to induce trends to improvement of health in at least one 
aspect of the life of users. For this purpose, both qualitative and quantitative factors were considered.

In terms of qualitative data, questionnaires were made to users at the end of the trials to get their feedback about how they perceived WellCo and if it motivated them to change.

In terms of quantitative data, statistics techniques were applied over the data available in the database with the purpose of getting objective data that supported the testimonies collected by qualitative questionnaires. Some of the elements assessed by this quantitative analysis were: steps taken, hours slept, quality of the nutrition consumed, mental health trends and interaction with WellCo app.

Along the following sections, the description and main conclusions derived from the qualitative and quantitative analysis of WellCo are collected. However, before disaggregating this information is important to clarify that, despite the promising results expected, lockdown and consecutive COVID waves made that the results of the project were not as good as initially expected.

\section{A. Qualitative Analysis}

The qualitative analysis aimed at assessing the experience of users with the model proposed by WellCo. The idea was being able to merge this information with the data coming from the quantitative analysis with the purpose of having relevant material to evaluate the results derived from the validation of the final prototype of WellCo for 4 months.

Due to the heterogeneity of users in every trial site (age, IT skills, area of population, etc), different qualitative questionnaires were created for users in Italy, Denmark and Spain. Although these questionnaires addressed the same topics, the idea was that they were adapted as much as possible to the context that surrounded each of these participants. Some of these topics were:

- User-friendliness and intuitiveness of the WellCo App;

- General functionality relevance;

- Health awareness/monitoring functionality;

- Specific WellCo app functionality;

- Virtual WellCo coach functionality and interaction;

- WellCo app questionnaires frequency and content;

- Overall expectations of the WellCo app pre- and post-participation;

- Overall assessment of the WellCo app.

Questions about each topic were defined by each pilot coordinators, adapting them to the context and characteristics of their population.

The questionnaires were carried out in different ways in the three countries: 1) in-deep interviews by phone; 2) online questionnaires (via email or WellCo app), 3) a mixture of both techniques, interviews and on-line questionnaires ${ }^{4}$. The organization of face-to-face interviews was discarded by all pilot's coordinators because of the health situation derived from Covid.

The results derived from the qualitative evaluation of the different countries shows the following:

\footnotetext{
${ }^{4}$ Online questionnaires were provided through

https://www.limesurvey.org, a professional survey platform.
}

- Danish users were the youngest and less motivated about WellCo. They were used to wear smartwatches to monitor their wellbeing and understand their own lifestyle. In addition, these users usually consumed Apple so the fact of providing WellCo as a native Android app was an important barrier to get these users motivated along the whole trial. Although they were sometimes sceptical about WellCo, they praised some aspects of WellCo such as the capability of WellCo to understand where they should improve as well as the information provided about sleep and physical activity.

- Spanish users were the keenest of WellCo solution, in fact, around $26 \%$ of them expressed their interest in continue using WellCo after the end of the project. This is a relevant percentage since these participants were the oldest and the one who had more limited ICT skills so the fact that more than a quarter of them showed motivation to continue using WellCo, even with the support of a trial coordinator, is very representative.

These users indicated that, in general terms, WellCo supposed a motivation to improve their wellbeing. They feel that recommendations meet their capability and opportunity to achieve goals and they assessed positively the fact of having supporting video-calls and the figure of an expert. Although they indicated that they feel motivated to achieve their goals, mainly because of the visualizations for progress monitoring, they did not show much enthusiasm for the Social Network. The reason is that most of these users lived in rural areas where everyone knows each other and they were reluctant to share their private life.

- Italian users expressed interest in the motivational approach followed by WellCo for daily lifestylerelated activities. They considered that the data about their activity served as stimulus to take measures and set improvement challenges. They also pointed out that the enthusiasm and satisfaction they felt when a goal was achieved, motivated them to keep improving and positively impacting on their lifestyle.

Thus, from the qualitative analysis is extracted that most users showed a positive attitude towards WellCo and the process for behaviour change. They recognized that WellCo impacted their lifestyle although this level of impact varied depending on variables such as age, type of living area (rural/urban) or other socio-economic factors such as the level of ICT skills or their current expenditure of technology.

Other important issue to remark is that during the trials, lockdown and repetitive COVID waves forced people to stay at home. This had an important effect in the WellCo results; aspects such as physical activity or sleep were considerably altered and special attention had to be put to other aspects such as mental status or nutrition. In addition, discouragement and fear to disease reduced the motivational level of people that relegated their concern for wellbeing to background. In this sense, strong emphasis was put to motivate people. To see in more detail, the impact COVID had on the wellbeing of people and the effort in motivation 
made by WellCo, in the following section information about the quantitative evaluation in WellCo is provided.

\section{B. Quantitative Analysis}

The quantitative analysis phase aimed at supporting or explaining with objective data, the statements made by participants during the quantitative assessment part. The quantitative process leveraged data about sleep, physical activity, nutrition, mental status and usage of WellCo data that was accessed via an API created for this purpose. This quantitative process compared this data in October, when the trials started and January, when the trials ended. The idea was to detect the percentage of users that showed improvement in one or several aspects of their wellbeing so the capacity of WellCo to impact the lifestyle of user was demonstrated.

Along the following section, a review of the main results extracted from the quantitative evaluation of each area affecting the wellbeing of users are provided.

\section{B.1. Physical Activity and Sleep Duration}

For the physical activity and sleep, two metrics were considered: physical activity in form of daily steps and sleep duration metric. Both metrics were acquired by the wearable devices worn by participants. The motivation for these metrics was as follows:

Physical inactivity factor is known to be one of the main causes, if not the major one, of chronic illness. Additionally, the focus on walking and overall activity in daily life reflected in daily steps, as opposite to focus on time-bound exercises like running or biking, has been established as a measure of physical activity in older populations. It is recommended for older adults to reach 7000-8000 steps a day to achieve their recommended daily activity levels.

Sleep, and especially sleep duration, is also an established factor contributing to the risk of chronic illness. It is recommended for adults to sleep 7-8 h (i.e., 420-480 minutes) per a day, to achieve their recommended daily sleep duration. It is recommended not to exceed $9 \mathrm{~h}$ of sleep (540 $\mathrm{min}$ ) on daily basis for best long term health results.

The results after the trials showed that a total of 41 users of 93 improved their steps from the beginning of trials till the end although not always achieving the recommendation (this could be because of the current mobility restrictions posed by COVID-19). Thus, a $44 \%$ of the total sample achieved an improvement in the number of steps. However, just a $32 \%$ of those who improved (13 of 41) achieved the recommendation of steps.

In relation to sleep, a total of 36 users improved their sleep from the beginning till the end of trials, although just 11 of them achieved their sleep recommendation. Then, it can be determined that around $39 \%$ of users (36 of 93) improved their sleep along the trials, although just a $31 \%$ (11 of 36) of them, met the sleep recommendation.

\section{B.2. Nutrition}

Related to nutrition, users' diet quality was assessed by assigning diet quality scores for nine goals - fruit intake, vegetables intake, fish intake, sugar intake, fat intake, protein intake, fibre intake, salt intake and water intake. To get the scores, users answered a food frequency questionnaire developed as part of the project[32]. This questionnaire had a frequency of two weeks.

The results indicate that more than $60 \%$ of the users in the sample improved their nutrition scores from the beginning till the end of the trials. The improvement happened in every nutrition category but salt intake.

\section{B.3. Mental Health}

For the assessment of the mental health, four states were considered: stress, anxiety, depression and general health. With mental health, WellCo aimed to cover episodes related to any of the above mentioned states with the purpose of providing recommendations that help to reduce or limited their frequency and impact.

To assess the effect of WellCo in mental health, the number of episodes and their severity determined by the mental health module at the beginning and at the end of the trials was determined.

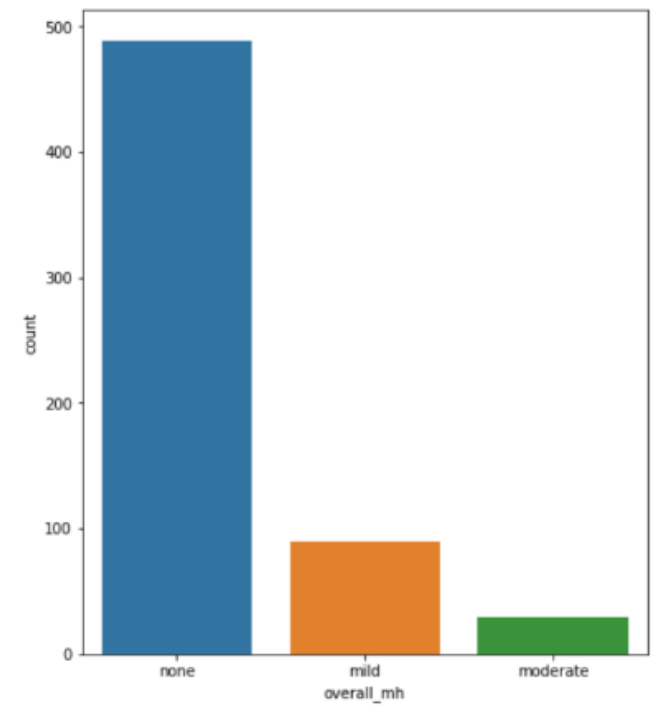

Fig. 7. Episodes of general mental health at trials beginning

As can be extracted from figure 7 and 8 , the number of "None" episodes have almost doubled from the beginning till the end of trials. Also, it can see how the number of mild episodes has passed from 90 at the beginning to around 40 in the case. For the case of moderate episodes have passed from 30 to 20 in M4, so the number of moderate mental health cases were reduced a $40 \%$. 


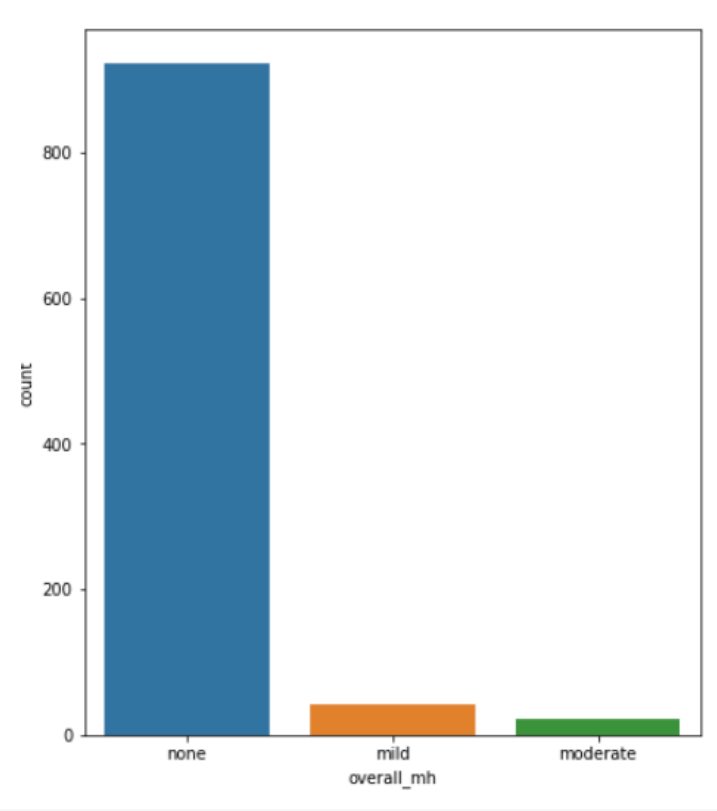

Fig. 8. Episodes of general mental health at trials end

\section{B.4. WellCo App Interaction}

Finally, to assess the impact of WellCo and the level of relation it had with the improvements described along the sections above, some measures aimed at evaluating the interaction of participants with WellCo along the duration of the trials were considered. These measures covered:

- Average number of milliseconds spent in the app per week along the duration of the trials. For the purpose of the analysis, this data was converted to minutes;

- Number of interactions with the coach per week along the duration of the trials. These interactions could be either to receive recommendations and goals or to ask something to the coach;

- Number of accesses to the activity section in the WellCo app per week along the whole duration of the trials;

- Number of goals the user achieved per week along the whole trial duration;

- Number of recommendations the user checked per week along the duration of the trials.

And the results showed that:

- A total of 91 users, $98 \%$, improved the time they spent in the app from the beginning of trials till the end. The time spent in the app per month was calculated as the sum of the minutes spent per week along the whole month.

- A total of 92 users, $99 \%$, increased the number of interactions with the coach since the beginning of the trials till the end, being the lowest value 10 interactions with the coach per month and the maximum, 38 interactions. The number of coach interactions per user per month has been calculated as the sum of interactions per week along the whole month.

- The number of accesses to the activity views (where users can see information about steps, sleep, heart rate and weight) also increased from the beginning to the end of the trials for all users.
The minimum of accessed corresponded to 16 and the maximum to 57 connections per month.

- Regarding the number of goals complete per month, along the whole duration of the trials, it was concluded than more than $89 \%$ of the users increased the number of goals complete from the beginning of the trials till the end. The maximum value corresponded to users with 7 goals achieved along the month (around 2 goals per week) and the minimum to 2 goals along the whole month.

- Finally, in relation to the number of recommendations monthly accessed by users, $\mathbf{9 2 \%}$ of users showed an increase in the number of recommendations accessed per month from the beginning to the end of the trials. The maximum value of access to recommendations is 70 , more than two recommendations per day, and the minimum is 16 .

Thus, from these results it can be concluded that there was an improvement in the interaction of the users with the WellCo app from the beginning till the end of the trials for every single feature measured although with some differences.

\section{CONCLUSIONS}

Along the whole paper, the method for behaviour change proposed by WellCo has been addressed as well as the main results derived from the validation with real users.

In terms of the model, WellCo leverages the main areas included in the behaviour change wheel model: capability, opportunity and motivation. It provides different functionalities and intelligence to detect the main areas where the user needs to improve as well as to ensure that behaviour change interventions meet these areas. In fact, one of the lessons learned from the project has been that motivation is the main area over which behaviour change interventions have to be built.

WellCo designed motivation over three main pillars: social incentives, immediate rewards and progress monitoring and continuous support; however, there are other aspects that have to be considered when offering an application for behaviour change. In the first place, is the type of population to address. If the population does not have IT skills, the application becomes a barrier that increase the fear of users about technology so motivation disappears and it becomes in rejection. Other important issue to consider is the kind of device that users currently wear - if in Denmark, where users mostly were Apple device, the project had provided WellCo in iOS format instead of Android, the motivation of user would have been different since the beginning.

In terms of validation, very promising results were obtained despite the lockdown and the COVID waves that took place during the project.

In areas such as physical activity or sleep, where the restrictions could have impacted more, almost $44 \%$ of the sample achieved an improvement in the number of steps and around $40 \%$ of users also improved their sleep during the trials. Even, the number of episodes related to mental health was reduced to halve since the beginning of the trials to the 
end. This was in fact, because of the special attention to motivation posed by WellCo that made that $98 \%$ increased the time they spent in the app from the beginning of trials to the end. Thus, instead of feeling demotivated as the trials evolved, users felt encouraged to continue using WellCo with more enthusiasm.

In this way, although some aspects have to be considered in future versions of WellCo to maximize its behaviour change potential, the results and lesson learned from the project are very promising and set the basis for higher research on this topic or future evaluations of the tool when the pandemic situation is calmed or disappeared.

\section{REFERENCES}

[1] Digital Transformation. Shaping the future of European Healthcare. Deloitte Center for Health Solutions. September 2020.

[2] Martínez, J. A. G., \& Estrada, A. F. (2020). Algunas recomendaciones de un modelo de referencia para la transformación digital en salud. Revista Cubana de Transformación Digital, 1(3), 100-111.

[3] Italy: Town pays people to cycle to work. Link: https://www.bbc.com/news/blogs-news-from-elsewhere34831650\#: : :text=An\%20Italian\%20town\%20will\%20pay,Il\%20Tirr eno\%20news $\% 20$ website\%20reports

[4] Dhingra, D., \& Dabas, A. (2020). Global Strategy on Digital Health. Indian pediatrics, 57(4), 356-358.

[5] Oliveira, J. S., Sherrington, C., Zheng, E. R., Franco, M. R., \& Tiedemann, A. (2020). Effect of interventions using physical activity trackers on physical activity in people aged 60 years and over: a systematic review and meta-analysis. British journal of sports medicine, 54(20), 1188-1194.

[6] Espín V, Hurtado MV, Noguera M. Nutrition for elder care: a nutritionalsemantic recommender system for the elderly. Exp Sys. 2016;33(2):201-10.

[7] Giabbanelli PJ, Crutzen R. Supporting self-management of obesity using anovel game architecture. Health Inform J. 2015;21(3):223-36.

[8] Bocanegra CLS, Ramos JLS, Rizo C, Civit A, Fernandez-Luque L. Healthrecsys:a semantic content-based recommender system to complement healthvideos. BMC Med Inform Decis Mak. 2017;17(1):

[9] Hidalgo JI, Maqueda E, Risco-Martín JL, Cuesta-Infante A, Colmenar JM,Nobel J. Glucmodel: a monitoring and modeling system for chronicdiseases applied to diabetes. J Biomed Inform. 2014;48:183-92

[10] Zhang W, Zou H, Luo L, Liu Q, Wu W, Xiao W. Predicting potential sideeffects of drugs by recommender methods and ensemble learning.Neurocomputing. 2016;173:979-87.

[11] Marlin BM, Adams RJ, Sadasivam R, Houston TK. Towards collaborativefiltering recommender systems for tailored health communications.American medical informatics association. In: AMIA annual symposiumproceedings; 2013. p. 1600

[12] Hors-Fraile, S., Schneider, F., Fernandez-Luque, L., Luna-Perejon, F., Civit, A., Spachos, D., ... \& de Vries, H. (2018). Tailoring motivational health messages for smoking cessation using an mHealth recommender system integrated with an electronic health record: a study protocol. BMC public health, 18(1), 1-10.

[13] Chen, Y. H., \& Sawan, M. (2021). Trends and Challenges of Wearable Multimodal Technologies for Stroke Risk Prediction. Sensors, 21(2), 460.

[14] Mohan, S., Thirumalai, C., \& Srivastava, G. (2019). Effective heart disease prediction using hybrid machine learning techniques. IEEE Access, 7, 81542-81554.

[15] Jiang, W., Wang, J., Shen, X., Lu, W., Wang, Y., Li, W., ... \& Chang, B. (2020). Establishment and validation of a risk prediction model for early diabetic kidney disease based on a systematic review and metaanalysis of 20 cohorts. Diabetes care, 43(4), 925-933.

[16] Zheng, Y., Hua, X., Win, A. K., MacInnis, R. J., Gallinger, S., Le Marchand, L., ... \& Newcomb, P. A. (2020). A new comprehensive colorectal cancer risk prediction model incorporating family history, personal characteristics, and environmental factors. Cancer Epidemiology and Prevention Biomarkers, 29(3), 549-557.

[17] Michie, S., Van Stralen, M. M., \& West, R. (2011). The behaviour change wheel: a new method for characterising and designing behaviour change interventions. Implementation science, 6(1), 1-12.
[18] Seshadri, D. R., Li, R. T., Voos, J. E., Rowbottom, J. R., Alfes, C. M., Zorman, C. A., \& Drummond, C. K. (2019). Wearable sensors for monitoring the physiological and biochemical profile of the athlete. NPJ digital medicine, 2(1), 1-16.

[19] Hagströmer, M., Oja, P., \& Sjöström, M. (2006). The International Physical Activity Questionnaire (IPAQ): a study of concurrent and construct validity. Public health nutrition, 9(6), 755-762.

[20] Buysse, D. J., Reynolds III, C. F., Monk, T. H., Berman, S. R., \& Kupfer, D. J. (1989). The Pittsburgh Sleep Quality Index: a new instrument for psychiatric practice and research. Psychiatry research, 28(2), 193-213.

[21] Jackson, C. (2007). The general health questionnaire. Occupational medicine, 57(1), 79-79.

[22] Robertson, D., Rockwood, K., \& Stolee, P. (1982). A short mental status questionnaire. Canadian Journal on Aging/La Revue canadienne du vieillissement, 1(1-2), 16-20.

[23] Gilbody, S., Richards, D., Brealey, S., \& Hewitt, C. (2007). Screening for depression in medical settings with the Patient Health Questionnaire (PHQ): a diagnostic meta-analysis. Journal of general internal medicine, 22(11), 1596-1602.

[24] Smerkol, M., \& Luštrek, M. (2018). Emotion Recognition Using Audio Speech Signal.

[25] Luštrek, M., Andova, A.(2019). Five attempts at cross-dataset speech emotion recognition.

[26] Gravenhorst, F., Muaremi, A., Bardram, J., Grünerbl, A., Mayora, O., Wurzer, G., ... \& Tröster, G. (2015). Mobile phones as medical devices in mental disorder treatment: an overview. Personal and Ubiquitous Computing, 19(2), 335-353.

[27] Kroenke, K., Spitzer, R. L., Williams, J. B., \& Löwe, B. (2009). An ultra-brief screening scale for anxiety and depression: the PHQ4. Psychosomatics, 50(6), 613-621.

[28] Warttig, S. L., Forshaw, M. J., South, J., \& White, A. K. (2013). New, normative, English-sample data for the short form perceived stress scale (PSS-4). Journal of health psychology, 18(12), 1617-1628.

[29] Luštrek, M., Fele, B., Reščič, N., \& Janko, V. (2019). Counting bites with a smart watch. In Slovenian Conference on Artificial Intelligence: proceedings of the 22nd International Multiconference Information Society-IS (pp. 49-52).

[30] Stankoski, S., Resçiç, N., Mezic, G., \& Lustrek, M. (2020, February). Real-time Eating Detection Using a Smartwatch. In EWSN (pp. 247252).

[31] Reščič, N., Valenčič, E., Mlinarič, E., Seljak, B. K., \& Luštrek, M. (2019, September). Mobile nutrition monitoring for well-being. In Adjunct Proceedings of the 2019 ACM International Joint Conference on Pervasive and Ubiquitous Computing and Proceedings of the 2019 ACM International Symposium on Wearable Computers (pp. 11941197).

[32] Reščič, N., Eftimov, T., Koroušić Seljak, B., \& Luštrek, M. (2020). Optimising an FFQ Using a Machine Learning Pipeline to teach an Efficient Nutrient Intake Predictive Model. Nutrients, 12(12), 3789.

[33] Wulfovich, S., Fiordelli, M., Rivas, H., Concepcion, W., \& Wac, K. (2019). "I Must Try Harder": Design Implications for Mobile Apps and Wearables Contributing to Self-Efficacy of Patients With Chronic Conditions. Frontiers in psychology, 10, 2388.

[34] Manea, V., \& Wac, K. (2018, October). mQoL: mobile quality of life lab: from behavior change to QoL. In Proceedings of the 2018 ACM International Joint Conference and 2018 International Symposium on Pervasive and Ubiquitous Computing and Wearable Computers (pp. 642-647).

[35] Laghouila, S., Manea, V., Estrada, V., \& Wac, K. (2018, April). Digital health tools for chronic illness and dementia risk assessment in older adults. In Annals of Behavioral Medicine (Vol. 52, pp. S291S291). JOURNALS DEPT, 2001 EVANS RD, CARY, NC 27513 USA: OXFORD UNIV PRESS INC.

[36] Mastropietro, A., Roecke, C., Porcelli, S., del Bas, J., Boquè, N., Maldonado, L. F., \& Rizzo, G. (2018, July). Multi-domain Model of Healthy Ageing: The Experience of the H2020 NESTORE Project. In Italian Forum of Ambient Assisted Living (pp. 13-21). Springer, Cham.

[37] Ricci, F., Rokach, L., \& Shapira, B. (2011). Introduction to recommender systems handbook. In Recommender systems handbook (pp. 1-35). Springer, Boston, MA.

[38] Sharot, T. (2017). What Motivates Employees More: Rewards or Punishments? Harvard Business Review. 


\section{Creative Commons Attribution License 4.0 (Attribution 4.0 International, CC BY 4.0)}

This article is published under the terms of the Creative Commons Attribution License 4.0

https://creativecommons.org/licenses/by/4.0/deed.en_US 